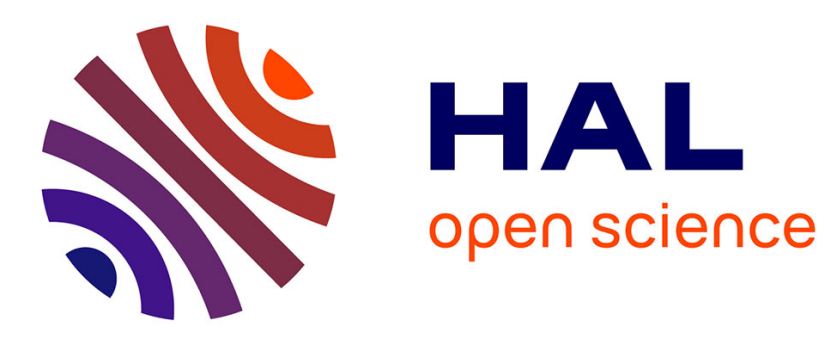

\title{
Hyperbolic Wavelet-Fisz denoising for a model arising in Ultrasound Imaging
}

Younes Farouj, Jean-Marc Freyermuth, Laurent Navarro, Marianne Clausel, Philippe Delachartre

\section{> To cite this version:}

Younes Farouj, Jean-Marc Freyermuth, Laurent Navarro, Marianne Clausel, Philippe Delachartre. Hyperbolic Wavelet-Fisz denoising for a model arising in Ultrasound Imaging. IEEE Transactions on Computational Imaging, 2017, 3 (1), pp.1-10. 10.1109/TCI.2016.2625740 . hal-01322246

\section{HAL Id: hal-01322246 https://hal.science/hal-01322246}

Submitted on 26 May 2016

HAL is a multi-disciplinary open access archive for the deposit and dissemination of scientific research documents, whether they are published or not. The documents may come from teaching and research institutions in France or abroad, or from public or private research centers.
L'archive ouverte pluridisciplinaire HAL, est destinée au dépôt et à la diffusion de documents scientifiques de niveau recherche, publiés ou non, émanant des établissements d'enseignement et de recherche français ou étrangers, des laboratoires publics ou privés. 


\title{
Hyperbolic Wavelet-Fisz denoising for a model arising in Ultrasound Imaging
}

\author{
Younes Farouj, Jean-Marc Freyermuth, Laurent Navarro, Marianne Clausel, and Philippe Delachartre
}

\begin{abstract}
We present an algorithm and its fully data-driven extension for noise reduction in ultrasound imaging. Our proposed method computes the hyperbolic wavelet transform of the image, before applying a multiscale variance stabilization technique, via a Fisz transformation. This adapts the wavelet coefficients statistics to the wavelet thresholding paradigm. The aim of the hyperbolic setting is to recover the image while respecting the anisotropic nature of structural details. The data-driven extension removes the need for any prior knowledge of the noise model parameters by estimating the noise variance using an isotonic Nadaraya-Watson estimator. Experiments on synthetic and real data, and comparisons with other noise reduction methods demonstrate the potential of our method at recovering ultrasound images while preserving tissue details. Finally, we emphasize the noise model we consider by applying our variance estimation procedure on real images.
\end{abstract}

Index terms - Hyperbolic wavelets, Fisz transformation, Variance stabilization, Gaussianization, Ultrasound imaging, Datadriven denoising.

\section{INTRODUCTION}

ULTRASOUND (US) imaging has been a well-established diagnostic tool in various medical applications for many years. This technology remains one the cheapest and safest medical imaging modalities. Nevertheless, the examination and the interpretation of ultrasound images is particularly challenging. This is mainly due to the presence of a particular type of noise called "speckle", which can also be found in similar imaging systems such as Synthetic-Aperture-Radar (SAR) and laser imaging. In ultrasound imaging, acquired signals are adjusted, inside the scanner, prior to display, by a non-linear processing transformation called log-compression [1]. This process aims at enhancing backscatterers in order to facilitate visual understanding. In this paper, our aim is to recover ultrasonic images using a relevant signal-dependent noise model [2] that takes into account the modification of noise characteristics due to the log-compression. Adaptations of non-local and variational techniques to this model have already been described in the literature respectively by Coupé et al. [3] then Jin and Yang [4].

Although these methods lead to good results in terms of signal-to-noise ratio, they still produce displeasing visual quality, mostly characterized by attenuated sharp edges. In this paper we present a different strategy which belongs to the wavelet denoising approaches [5]. As in the majority of denoising approaches, the wavelet denoising paradigm relies on the limiting assumption that the noise is additive white Gaussian noise (AWGN). To go beyond this case, we adapt a multiscale variance stabilization technique introduced by Fryzlewicz [6] in order to make the distribution of wavelet coefficients asymptotically Gaussian with the same variance. We extend this method to hyperbolic wavelets and show how variance stabilization can be easily performed using the low frequency outputs from the wavelet transform at different scales. The motivation behind the use of hyperbolic wavelets is their capacity to provide better estimators than the standard wavelet-tensor construction whenever images contain anisotropic features [7][8]. This situation often occurs in ultrasound imaging due to the presence of features skin layers, vessels. Finally, we show how our approach can be performed in a blind mode, that is, without any prior knowledge of the noise variance parameters. This involves the use of a mean filter for a pre-estimation of the image. The noise component is then computed using a Nadaraya-Watson estimator.

To validate our methods we present numerical experiments based on synthetic and real data, and comparisons with the state of the art non-local and variational algorithms. We demonstrate that our data-driven approach performs nearly as well as in situations in which the noise variance is known. Moreover, the measured variance on real ultrasound images confirms the relevance of the noise model we are considering.

The rest of the paper is organized as follows. A brief overview of different US noise models and dedicated denoising techniques is presented in Section II. In section III, we describe our novel wavelet based methodology. Finally, we provide extensive experimental results and comparisons in Section IV.

This work was supported by "Région Rhône-Alpes" under the ARC 6. M. Clausel's research is supported by the French Agence Nationale de la Recherche (ANR) under reference ANR-13-BS03-0002-01 (ASTRES) and PERSYVAL-Lab (ANR-11-LABX-0025-01). L. Navarro's research is supported by the (ANR) under reference ANR-15-CE19-0002 (LBSMI). P. Delachartre is within the framework of the Labex CELYA (ANR-10-LABX-0060) of the Universite de Lyon. J.-M. Freyermuth's research was supported by the Engineering and Physical Sciences Research Council [EP/K021672/2]

Y. Farouj and P. Delachartre are with the Université de Lyon; CREATIS; CNRS UMR5220; Inserm U1044; INSA-Lyon; Lyon 69622, France (e-mail: farouj@creatis.insa-lyon.fr; delachartre@creatis.insa-lyon.fr).

J.-M Freyermuth is with the Statistical Laboratory, DPMMS, University of Cambridge, Cambridge, CB3 0WB (e-mail: jmf84@cam.ac.uk).

L. Navarro is with the Ecole Nationale Supérieure des Mines; CIS-EMSE; CNRS UMR 5307; LGF, F-42023 Saint-Etienne, France (e-mail: navarro@emse.fr).

M. Clausel is with the Université de Grenoble; Laboratoire Jean Kuntzmann; CNRS UMR 5224, Grenoble, France (e-mail: marianne.clausel@imag.fr). 


\section{RELATED WORK}

Novel techniques emerging in the general image processing community have been continuously adapted to deal with US noise models. Hereafter, we propose an overview of the main models and techniques we are concerned with.

\section{A. Multiplicative Noise}

An important challenge in the process of denoising ultrasound images is to find an adequate noise model. The noise can be described as the sum of individual scattered beams from tissue inhomogeneities. Hence, one can derive a natural noise model from the knowledge of the statistics of the echo signal. It has been shown that the probability density function of the scatters is a Rayleigh distribution [1]. This understanding gave rise to multiplicative noise models similar to those used in SAR imaging. Many filters have been proposed for such a model, including the seminal works by Lee [9], Frost et al.[10] and Kuan [11]. Anisotropic diffusion filters [12] have also been successful in US imaging. These include adaptations to account for speckle noise statistics as in the Speckle Reducing Anisotropic Diffusion (SRAD) [13] and its oriented version (OSRAD) [14], and more recently, memory-driven filters [15].

\section{B. Additive Noise}

Multiplicative noise models do not take into consideration the image formation process leading to the final US images visualized on scanners. In particular, a process called logarithmic compression [1] is used to enhance backscatterers suffering from low contrast in order to improve visual understanding. A simple solution is to assume that the signal and the noise are totally distinct. Thus the logarithmic compression step transforms the multiplicative noise into an additive signal-independent model

$$
v=u+\varepsilon
$$

where $v$ is the observation, $u$ is the unknown image and $\varepsilon$ is the noise component. Assuming that $\varepsilon$ is a zero-mean Gaussian white noise $\varepsilon \sim \mathcal{N}\left(0, \sigma^{2}\right)$, with $\sigma \in(0, \infty)$ leads to AWGN models widely studied in signal and image processing. In particular, these models are well suited for wavelet thresholding [5] and have been used for US applications [16]. Achim et al.[17] showed that under model (1), the wavelet coefficients of the noise, after logarithmic transformations, have non-Gaussian statistics which can be described by some alpha-stable distributions [18].

\section{Hybrid Noise}

The main drawback of model (1) is the violation of the assumption that the noise is correlated with underlying tissues. This assumption is widely used and accepted in Echography. For example, it is the key idea behind motion estimation via speckle tracking [19]. It turns out that the logarithmic compression makes the statistics of ultrasound images deviate from the Rayleigh distribution [20]. A more relevant model for ultrasound noise was presented in [2] and assumes that the variance of the noise component is no longer constant but respects the following equation

$$
v=u+u^{\gamma} \varepsilon
$$

where $\varepsilon$ is a Gaussian noise as in (1) and $\gamma>0$. Model (2) seems to be more appropriate as it preserves the signal dependency and has shown been to be effective for speckle modelling [2], as well as motion estimation in US image sequences [21]. In this paper, we develop an appropriate wavelet thresholding method assuming model (2) holds true. Adaptations of two other classical paradigms in denoising, beside wavelet methods, have been studied for model (2) [3][4]. We recall these paradigms:

Nonlocal methods. The non local point of view was initially developed in [22] leading to the famous N-L means filter. It can be seen as a type of smart average filtering that uses the fact that similar pixels are not necessarily neighbours. Given two pixels, the similarity measure is the Euclidean distance between patches within their respective neighborhoods. As the Euclidean distance is more appropriate in white noise cases [22]. Coupé et al. [3] presented the OBNLM algorithm in which the Pearson distance is used along with an optimized version of the N-L means filter.

Variational methods. The variational approach relies on the minimization of a functional involving a data-fidelity term and a regularity assumption. A common assumption is that images belong to BV (Bounded Variation) spaces, and so Total Variation (TV) is often used for the regularization term [23]. For AWGN models, the fidelity term is simply given as the Euclidean distance between the unknown image and the corrupted one. Adaptations to model (2) consists in dividing the fidelity term by the unknown image to the power $\gamma$, which is also the standard deviation of the noise. The reader is referred to papers by Rudin, Lions and Osher for the case $\gamma=1$ [24] and by Jin and Yang for the case $\gamma=0.5$ [4]. However, the functional cannot be minimized via simple primal dual algorithms [25] as in the AWGN case, a gradient descent is required.

In the following section we propose a technique to adapt the wavelet-based methods to the noise model (2). Moreover, we present a data-driven algorithm which solves the problem without prior knowledge of the parameters $\sigma$ and $\gamma$ of model (2). 


\section{METHOD}

Hyperbolic wavelet bases are unconditional bases for functions in $L^{2}\left([0,1]^{2}\right)$. They provide sparse representations so that the simple hard thresholding procedure which consists in keeping only coefficients with magnitude larger than a given threshold; setting the others to zero, provide estimators with very good theoretical and practical performances [7][8].
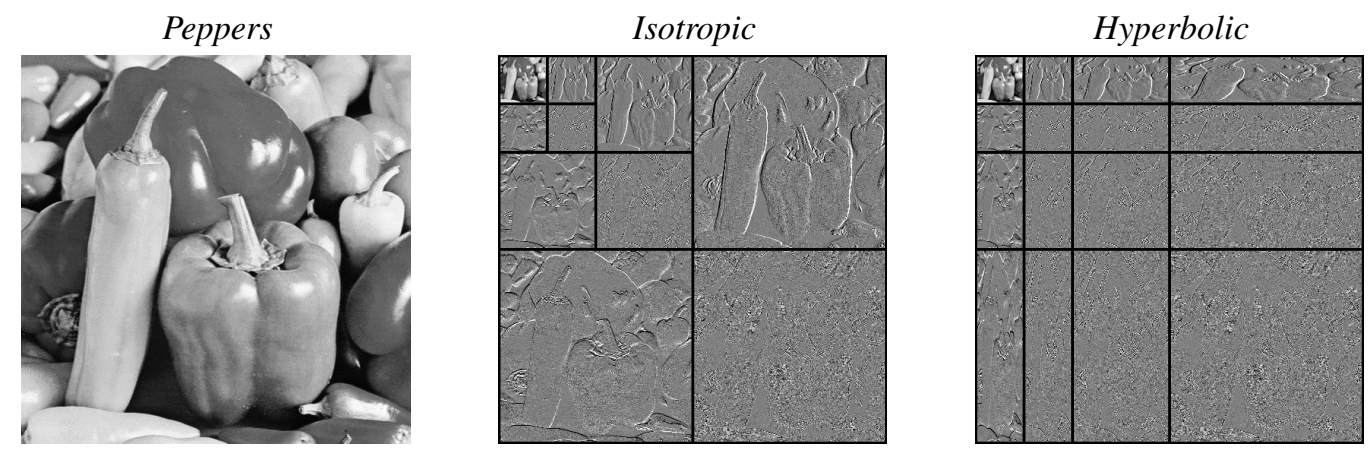

Figure 1: Wavelet decomposition in isotropic and hyperbolic settings

\section{A. Notations}

We begin with the hyperbolic wavelet transform (HWT). The starting point is a one-dimensional function $\psi$, called the mother wavelet, to which one can associate dilated and translated versions $\psi_{j, k}()=.2^{j / 2} \psi\left(2^{j} .-k\right)$ with $j \geq 0$ and $k \geq 0$. In the same manner, a scaling function $\varphi$ is defined, along with its dilated and translated versions $\varphi_{j, k}()=.2^{j / 2} \varphi\left(2^{j} .-k\right)$. Then, the $2 \mathrm{D}$ HWT basis of $L^{2}\left([0,1]^{2}\right)$ is given by

$$
\begin{aligned}
\psi_{j_{1}, j_{2}, k_{1}, k_{2}}\left(x_{1}, x_{2}\right) & =\psi_{j_{1}, k_{1}}\left(x_{1}\right) \psi_{j_{2}, k_{2}}\left(x_{2}\right), \\
\psi_{0, j_{2}, k_{1}, k_{2}}\left(x_{1}, x_{2}\right) & =\varphi_{0, k_{1}}\left(x_{1}\right) \psi_{j_{2}, k_{2}}\left(x_{2}\right), \\
\psi_{j_{1}, 0, k_{1}, k_{2}}\left(x_{1}, x_{2}\right) & =\psi_{j_{1}, k_{1}}\left(x_{1}\right) \varphi_{0, k_{2}}\left(x_{2}\right), \\
\psi_{0,0, k_{1}, k_{2}}\left(x_{1}, x_{2}\right) & =\varphi_{0, k_{1}}\left(x_{1}\right) \varphi_{0, k_{2}}\left(x_{2}\right),
\end{aligned}
$$

for all $\left(j_{1}, j_{2}\right) \in \mathbb{N} \times \mathbb{N}$ and $\left(k_{1}, k_{2}\right) \in \mathbb{Z}^{2}$. This construction differs from that of the classical two dimensional Discrete Wavelet Transform (DWT), in the sense that different dilation factors are used in each dimension. In the case of the standard 2D DWT, only the cases $j_{1}=j_{2}$ are allowed; therefore the resulting atoms are isotropic.

Let us note $I=\left\{j=\left(j_{1}, j_{2}\right) \in \mathbb{N}^{2}\right.$ and $\left.\underline{k}=\left(k_{1}, k_{2}\right) \in \mathbb{Z}^{2}\right\}$ The projection of a function $f$ of $L^{2}\left([0,1]^{2}\right)$ onto the HWT basis gives a set of hyperbolic wavelet coefficients $\left\{d_{\underline{j}, \underline{k}}\right\}_{(\underline{j}, \underline{k}) \in I}$ where

$$
d_{\underline{j}, \underline{k}}(f)=\left\langle\psi_{\underline{j}, \underline{k}}, f\right\rangle .
$$

The set $\left\{d_{\underline{0}, \underline{k}}\right\}$, where $\underline{0}=(0,0)$, represent the approximation coefficients. In finite discrete settings, a maximal scale is fixed at $J=\log _{2}(N)$ for an image of size $N \times N$. As the HWT can be seen as a tensor product of one-dimensional wavelet transforms, the numerical implementation of the HWT can be achieved by applying two successive 1D DWT on each of the two dimensions. Figure 1 highlights the difference of the scale-space tilling in the standard and hyperbolic settings.

\section{B. Wavelet denoising}

When the noisy observation $v$ verifies model (1), the very simple, but powerful, procedure of wavelet thresholding mentioned earlier can be used. In the wavelet domain, the additive model (1) reads

$$
d_{\underline{j}, \underline{k}}(v)=d_{\underline{j}, \underline{k}}(u)+d_{\underline{j}, \underline{k}}(\varepsilon),
$$

with $(\underline{j}, \underline{k}) \in I$. The idea behind wavelet methods comes from the efficiency of wavelet coefficients in the sparse representation of images. A simple process consists in constructing an estimator $\hat{u}_{\sigma}$ given by

$$
\hat{u}_{\sigma}=\sum_{(\underline{j}, \underline{k}) \in I_{\sigma}} d_{\underline{j, k} \underline{k}}(v) \psi_{\underline{j}, \underline{k}}
$$


where $I_{\sigma}=\left\{(j, \underline{k}) \in I\right.$, such that $\left.\left|d_{j, k}(v)\right|>t(\sigma)\right\}$ and $t(\sigma)$ is the threshold parameter. This will basically remove the noisy coefficients. Moreover, one of the distinctive features of this elitist empirical algorithm is the existence of a universal threshold given by

$$
t(\sigma)=\sqrt{2 \log (\operatorname{Card}(I)) \operatorname{Var}\left(d_{\underline{j}, \underline{k}}(\varepsilon)\right)}=\sigma \sqrt{2 \log \left(N^{2}\right)},
$$

In image restoration, it is often considered that the unknown image belongs to spaces with regularity varying along the two dimensions. This notion of anisotropy is at the heart of multivariate function estimation [7]. Hyperbolic wavelets are well suited to such situations [27]. It has recently been shown [28] that mixing scales when constructing wavelets, as in (3), makes thresholding techniques comparable to state-of-the art denoising algorithms. Still, the choice of the threshold (7) is crucial, and its existence relies on the fact that the wavelet coefficients of the noise are Guassian and independent. In the next section we show how, in the case of the ultrasound noise model (2), this obstacle can be overcome via a wavelet-based variance stabilization technique.

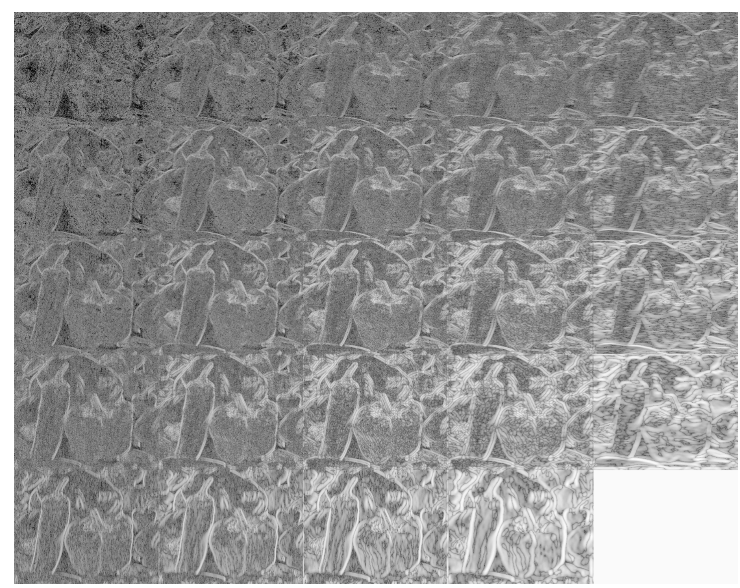

(a)

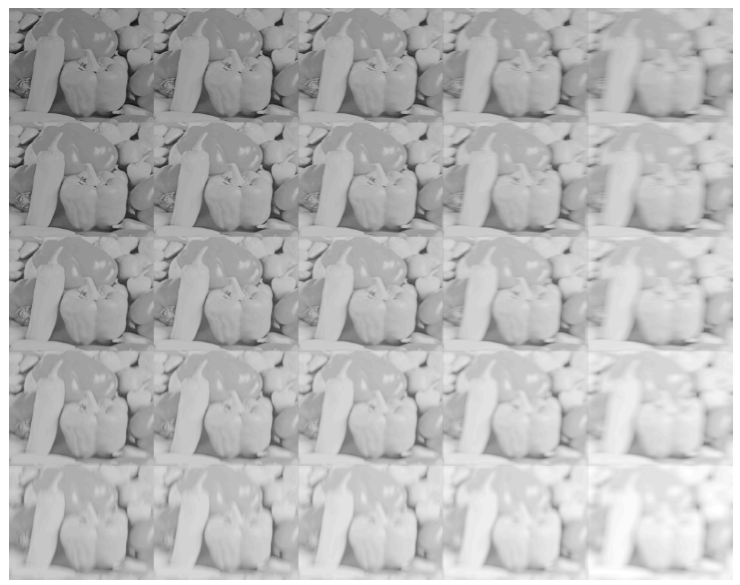

(b)

Figure 2: Outputs of the hyperbolic NDWT of the "Peppers" image: (a) The set of wavelet coefficients $\left\{d_{j, \underline{k}}\right\}_{j, \underline{k}}$ and (b) the set of approximation coefficients $\left\{c_{\underline{j}, \underline{k}}\right\}_{\underline{j}, \underline{k}}$

\section{The Proposed Wavelet-Fisz (WF) approach}

In (2), the noise component is of the form

$$
\eta=u^{\gamma} \varepsilon,
$$

thus, its variance depends on the unknown image. In order to obtain an adaptive image-dependent threshold we extend results from [6] to the two-dimensional case while adapting it to our problem.

Lemma 3.1: Let $\left\{\psi_{\underline{j}, \underline{\underline{k}}}\right\}_{(\underline{\underline{j}}, \underline{\underline{k}}) \in I}$ be a normalized wavelet basis, in the sense that $\|\psi\|_{2}^{2}=1$. Let $u_{\underline{j}, \underline{\underline{k}}}$ denote the restriction of $u$ in the support of the function $\psi_{\underline{j}, \underline{k}}$. Assume that, for each $(\underline{j}, \underline{k}) \in I, u_{\underline{j}, \underline{k}}$ can be approximated by a constant function $\bar{u}_{\underline{j}, \underline{k}}$. Then we have

$$
\left(\frac{d_{\underline{j}, \underline{k}}(\eta)}{\sigma \bar{u}_{\underline{j}, \underline{k}}^{\gamma}}\right)_{\underline{j}, \underline{k}} \sim \mathcal{N}(0,1) \text {. }
$$

In words, lemma 3.1 guarantees that the vector $\left(\frac{d_{j, \underline{k}}(\eta)}{\bar{u}_{\underline{j}, \underline{k}}^{\gamma}}\right)_{j, \underline{k}}$ is asymptotically normal, in the distribution sense, when $u_{\underline{j}, \underline{k}}$ is approximated by $\bar{u}_{j, \underline{k}}$. In Appendix A, we derive the variance given in (9). Convergence results are immediate from the one-dimensional case [6]. The idea of applying the Gaussianizing routine to wavelets coefficients was first introduced for Poisson intensity estimation by Fryzlewicz and Nason [29], following a general framework introduced by Fisz [30] ${ }^{1}$. It was later extended to Poisson intensity estimation in images [31]. An approximation $\bar{u}_{j, \underline{k}}$ of the unknown image $u$ needs to be computed in the support of the function $\psi_{\underline{j}, \underline{k}}$. A key point here is our use of the low frequency outputs of the wavelet transform at each scale as local means pre-estimations. These outputs are given by scaling coefficients:

$$
c_{\underline{j}, \underline{k}}(f)=\left\langle\varphi_{\underline{j}, \underline{k}}, f\right\rangle
$$

${ }^{1}$ Hence the name Wavelet-Fisz. 
where

$$
\varphi_{j_{1}, j_{2}, k_{1}, k_{2}}\left(x_{1}, x_{2}\right)=\varphi_{j_{1}, k_{1}}\left(x_{1}\right) \varphi_{j_{2}, k_{2}}\left(x_{2}\right) .
$$

The support of the function $\varphi_{j, \underline{k}}$ decreases as the value $|\underline{j}|=j_{1}+j_{2}$ decreases. As a consequence of the law of large numbers, the local means approximation (10) becomes less accurate. This issue has limited consequences since, following [6], we consider only the coarsest scales up to a certain level $|j| \leq J_{\max }$. Not much information is lost since the finest scales consist of high-frequency components which are essentially noise. Using the lemma 3.1, we can now define a new set for the construction of the nonlinear estimator (6) given by

$$
\tilde{I_{\sigma}}=\left\{(\underline{j}, \underline{k}) \in I, \text { s.t }|\underline{j}| \leq J_{\max } ; \frac{\left|d_{\underline{j}, \underline{k}}(v)\right|}{\sigma c_{\underline{j}, \underline{k}}(v)^{\gamma}}>t(1)\right\} .
$$

Implementation: The presented WF technique can be performed using a non-decimated wavelets transform (NDWT). The wavelets coefficients (4) and the approximation coefficients (10) for the NDWT are presented in Figure 2. It has been shown that the denoising methods based on NDWT outperforms those based on traditional (decimated) wavelets in terms of mean-squared error (MSE) and signal-to-noise ratio (SNR) [32]. This is mainly due to its translation invariance. However, the non-decimated wavelet coefficients are, in general, correlated even if the noise is independent. The choice of relevant wavelet coefficients becomes a correlated multiple hypothesis testing problem. Thus, the choice of the threshold (7) can lead to non-optimal results. In practice, one can consider the non-decimated wavelet coefficients as separate packets of uncorrelated coefficients [33]. The universal threshold can then be applied to each packet. The pseudo code for the routine is given in Algorithm 1.

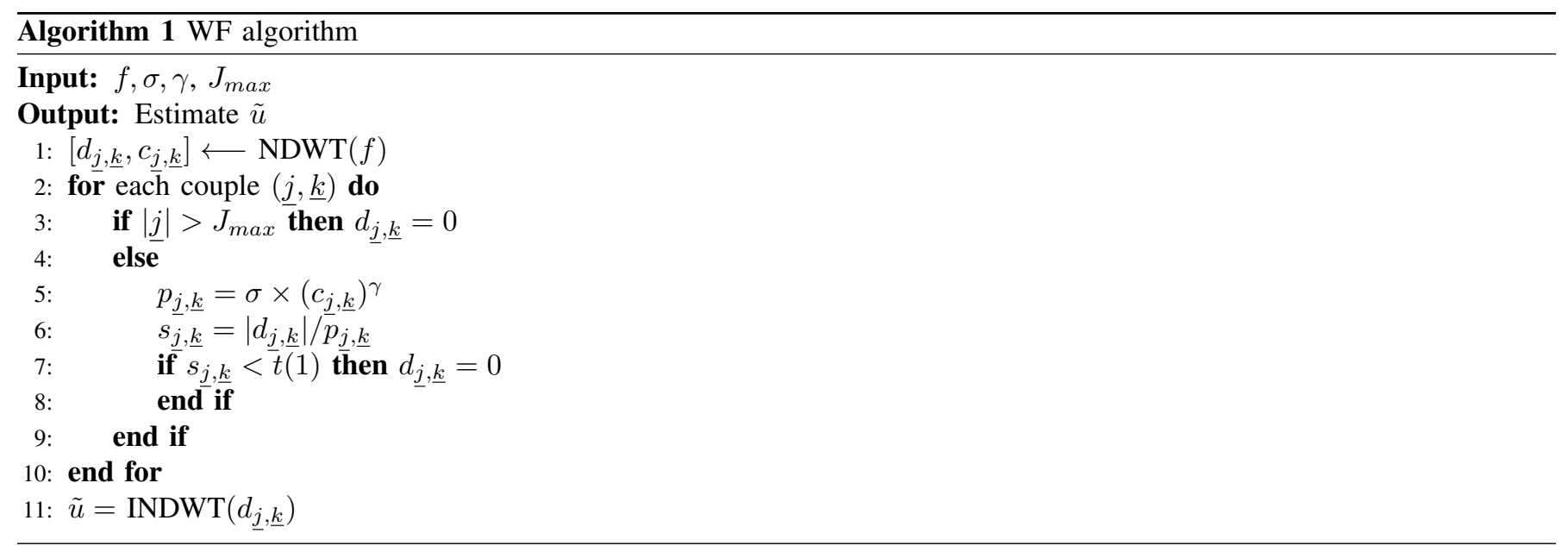

The key step in this algorithm is the stabilization technique leading to the set $\left(s_{j, k}\right)_{(j, k) \in I}$. Figure 3 shows how the wavelet coefficients are stabilized after the WF procedure. Here, we used the stabilization technique with $\sigma=2$ and $\gamma=0.5$. The wavelet coefficients magnitudes were scaled beeteween 0 and 1 and a normal distribution fitting algorithm was applied. The Liver image represents a section of a human liver along with the portal vein. The diagonal details of the wavelet transform at the first thresholding scale are examined. At fine scales, the wavelet transformation retrieves, mainly, the noise component. We can clearly see that the distribution of the wavelet coefficients deviates from the Gaussian distribution. This phenomenon can be explained by model (2) as the noise is perturbed by the image statistics. In fact, it was observed that the statistics of the wavelet coefficients of an image are more likely to follow distributions with heavier tails than a Gaussian one, such as Exponential and Laplacian distributions [26][34]. Note that the non-Gaussianity of the wavelet coefficients distribution in US images was first observed by Achim et al. [17]. In this latter work, the authors assumed that the noise has an alpha-stable distribution.

\section{Fully data-driven extension}

Besides the fact that there is no conventional noise model in ultrasound imaging, different authors may use different parametrizations for a given noise model. In particular, for our model of interest, different values for the parameters $\sigma$ and $\gamma$ are given in [3],[4] and [24]. A point of debate is whether a large value should be used for $\gamma$ and a small one for $\sigma$ or vice versa. We sidestep the problem by estimating the standard deviation of the noise directly from the data.

1) Standard deviation estimation: To address this problem, any filter of low computational cost can be used on the noisy image to obtain a pre-estimation $\bar{u}$. We applied a simple mean filter of size $M$ to the image. An estimation of the noise component $\eta$ is then given by the residual

$$
\hat{\eta}(\bar{u})=v-\bar{u}
$$


Liver

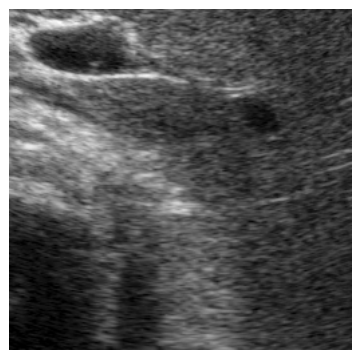

Coefficients distribution

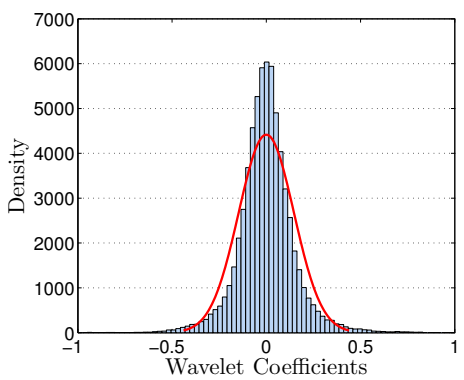

Stabilized coefficients distribution

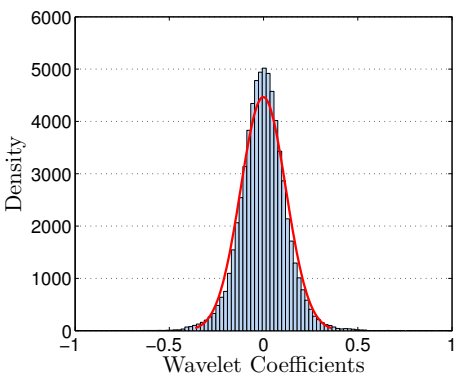

Figure 3: Wavelet decomposition of US images: Statistics of the diagonal details at the finest scale

To estimate the variance, a kernel smoothing technique is applied to the highly oscillating squared residuals $\hat{\eta}^{2}$. For any vector $w$ with values belonging to $[\min (\bar{u}), \max (\bar{u})]$, the variance estimator of $w$ is given as

$$
h(w)=\widehat{\operatorname{Var}(\eta(w)})=\frac{\left\langle\hat{W}_{b}(w), \hat{\eta}^{2}\right\rangle}{\hat{W}_{b}(w)},
$$

where $W$ is defined as

$$
\hat{W}_{b}(w)=\frac{1}{N^{2} b} K\left(\frac{\bar{u}-w}{b}\right),
$$

with $b$ the bandwidth of the kernel $K$. This regression technique is called the Nadaraya-Watson estimation. Under the assumption that the variance of the noise is a positive power of the image intensity, as suggested by model (2), it is natural to constraint the estimator of the variance to be non-decreasing. This can be done using the so-called isotonic regression, which consists in finding the closest non-decreasing function, in term of least mean square error, using a "pool-adjacent-violators" algorithm [35]. We present an example of such a routine on a corrupted Peppers image. Our choice of the image Peppers is motivated by the fact that it has many variations in grey values, resulting in an interval of intensities well covered by the vector $w$. The results of two experiments with different values of $\gamma$ and $\sigma$ are given in Figure 4. As the image pixel values range from 0 to 255 , we simply choose $w$ to be a uniform discretization of $[0,255]$. The size of the average filter depends on the homogeneity of the image, in this example we used $M=12$. A Gaussian kernel with a bandwidth $b=1$ was used for $K$. The results confirm the reliability of the standard deviation estimator $\sqrt{h}$ in comparison to the ground truth.
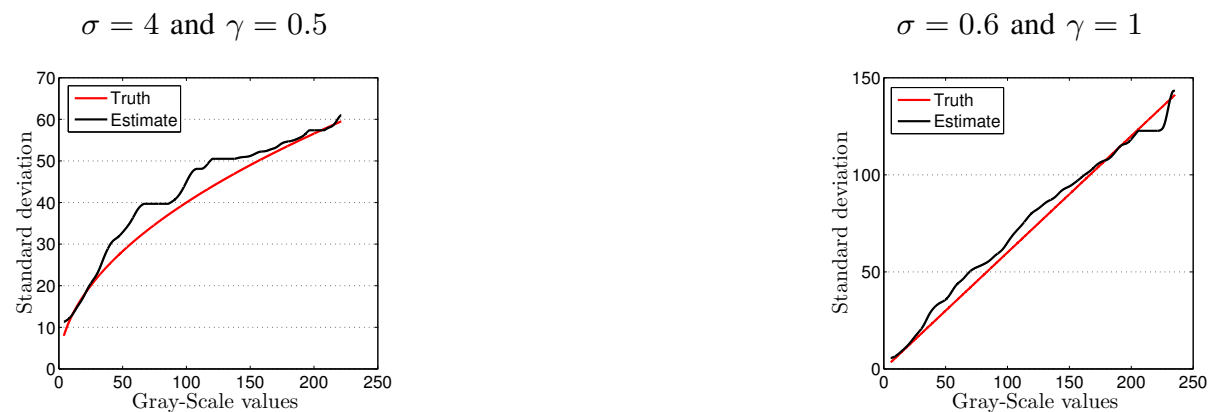

Figure 4: Standard deviation estimation from experiments on the "Peppers" image for different values of $\sigma$ and $\gamma$.

2) Blind denoising: We describe the adaptation of the WF algorithm to the fully data-driven methodology. For our noise model, the standard deviation estimator presented in III-D1 gives the following approximation

$$
\sqrt{h(w)} \approx \sigma w^{\gamma},
$$

hence, we have a similar result to the one given in lemma 3.1

$$
\left(\frac{d_{\underline{j}, \underline{k}}(\eta)}{\sqrt{h\left(c_{\underline{j}, \underline{k}}\right)}}\right)_{\underline{j}, \underline{k}} \sim \mathcal{N}(0,1),
$$

To remove the parameters $\sigma$ and $\gamma$ from the WF algorithm, the computation of the auxiliary step 5 is changed to

$$
p_{\underline{j}, \underline{k}}=\sqrt{h\left(c_{\underline{j}, \underline{k}}\right)},
$$




\section{EXPERIMENTS AND DISCUSSION}

In this section we present some experiments to evaluate the performance of our WF method. In order to distinguish the different contributions of our work, we divide this section into two parts. First, we focus on the performance of the WF method in both Isotropic (IWF) and Hyperbolic (HWF) settings, and then we show the potential of the data-driven extension.

\section{A. The WF method}

We compare our results to those obtained using two other approaches that considers the noise model given by (2). The OBNLM filter have proven to be very effective in speckle noise reduction and to perform better than classical filters [3]. The variational approach [4] is an adaptation of the well-established TV denoising to model (2). The criteria used for the comparisons were the classical Peak Signal to Noise Ratio (PSNR), and the Structural Similarity Index Measure (SSIM) [36] which allowed evaluation of the tissue structure preservation. As the great majority of ultrasound imaging is not concerned with functional studies, the preservation of morphological information while performing denoising is normally more important than preserving the true measured pixel intensity. We also show the difference between the true image and the denoised result of every method. This is known in the literature as the method noise [22]. One except to retrieve more noise in areas of high pixel intensities according to model (2). The OBNLM filter is available on the web ${ }^{2}$. The parameters $\alpha$ and $M$ controlling the number of blocks and the size of the search window are fixed at 3 and 6 as in the original paper, and the filtering parameter $h$ was optimized for different levels of noise. The variational algorithm was implemented with the gradient descent step fixed at 0.2 as suggested by the authors. We use Haar wavelets for the WF method. The scaling function associated to these wavelets behave like a simple mean filter which gives a reliable set of approximation coefficients $\left\{c_{j, \underline{k}}\right\}_{j, \underline{k}}$. These wavelets are also efficient at preserving discontinuities. We are aware, however, that these wavelets do not lead to optimal results in terms of PSNR and its is possible to improve the results using wavelets from other families such as Daubechies or Coiflets. In all experiments, the coefficients corresponding to the first finest scale are truncated.

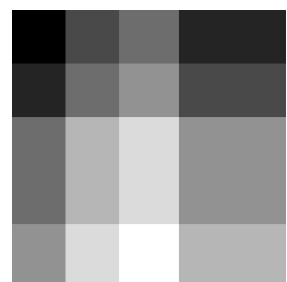

Blocks

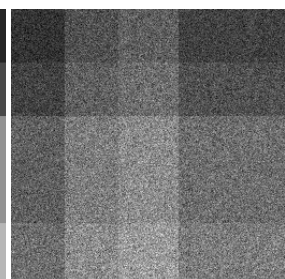

Noisy $(\sigma=4)$

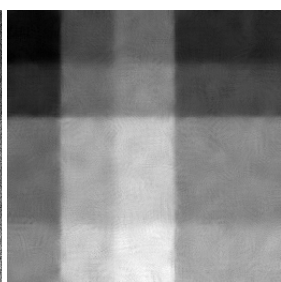

OBNLM

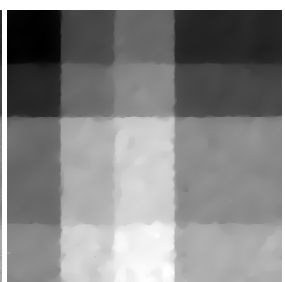

Variational

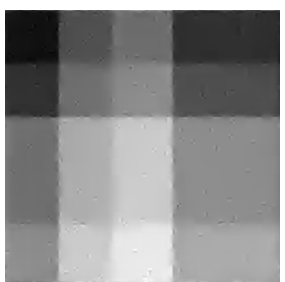

IWF

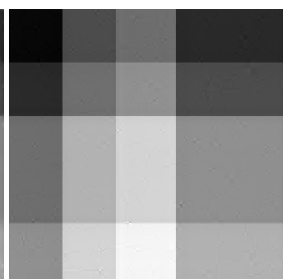

HWF

Figure 5: Results of various methods applied to the image Blocks. Quantitative evaluation is given in Table I.

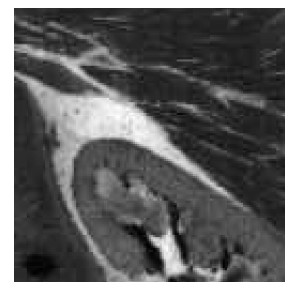

Kidney

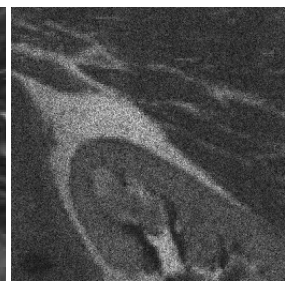

Noisy $(\sigma=4)$

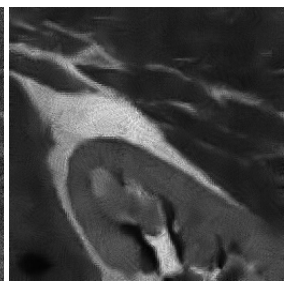

OBNLM

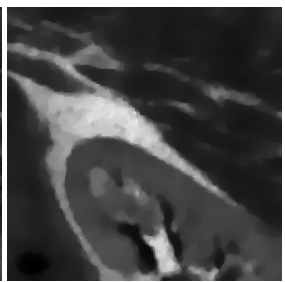

Variational

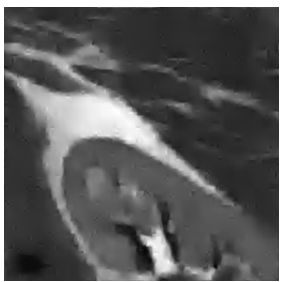

IWF

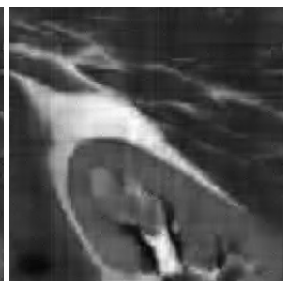

HWF

Figure 6: Results of various methods applied to the image Kidney. Quantitative evaluation is given in Table I.

1) Experiments on synthetic data: Our two Synthetic experiments were performed by adding synthetic noise to clean images. We fix $\gamma=0.5$, as in [3] and [4]. The first image Blocks aims only at demonstrating the ability of hyperbolic wavelets to deal with highly anisotropic images. In fact, this image is an additive model when the regularities in the two space dimensions are independent. This is a highly anisotropic case where the hyperbolic setting gives optimal results [8]. The second Kidney image is a CT image taken from FIELD II's website ${ }^{3}$. This latter example is challenging to denoise because of the presence of many gray level variations.

Table I outlines the performances of the different methods together with optimal parameters. In the case of the image Blocks, the contribution of the hyperbolic setting is clearly visible in terms of PSNR, SSIM and visual quality (e.g. Figure 5). The hyperbolic Wavelet-Fisz thresholding gives the best results for all noise levels. The variational approach also gives good results

\footnotetext{
${ }^{2}$ https://sites.google.com/site/pierrickcoupe

${ }^{3}$ http://field-ii.dk
} 


\begin{tabular}{lccc|ccc}
\hline & \multicolumn{3}{c|}{ Blocks } & \multicolumn{3}{c}{ Kidney } \\
\cline { 2 - 7 } & PSNR $(\mathrm{dB})$ & SSIM & Parameters & PSNR (dB) & SSIM & Parameters \\
Noisy $(\sigma=2)$ & 22.83 & 0.135 & - & 24.83 & 0.430 & - \\
OBNLM & 35.13 & 0.917 & $h=1.5$ & 30.05 & 0.845 & $h_{=1}$ \\
Variational & 37.98 & 0.972 & $n_{\text {iter }}=180$ & 28.72 & 0.814 & $n_{\text {iter }}=160$ \\
IWF & 35.78 & 0.958 & - & 29.04 & 0.837 & - \\
HWF & $\mathbf{4 9 . 6 5}$ & $\mathbf{0 . 9 9 3}$ & - & $\mathbf{3 0 . 2 4}$ & $\mathbf{0 . 8 6 6}$ & - \\
& & & & & \\
Noisy $(\sigma=3)$ & 20.95 & 0.071 & - & 22.30 & 0.272 & - \\
OBNLM & 32.86 & 0.836 & $h=2$ & $\mathbf{2 8 . 7 1}$ & 0.752 & $h=1$ \\
Variational & 35.57 & 0.955 & $n_{\text {iter }}=260$ & 27.91 & 0.799 & $n_{\text {iter }}=180$ \\
IWF & 33.75 & 0.929 & - & 27.39 & 0.791 & - \\
HWF & $\mathbf{4 6 . 6 5}$ & $\mathbf{0 . 9 8 7}$ & - & 28.20 & $\mathbf{0 . 8 2 2}$ & - \\
& & & & & \\
Noisy $(\sigma=4)$ & 19.31 & 0.044 & - & 20.83 & 0.187 & - \\
OBNLM & 31.38 & 0.739 & $h=2.5$ & 27.81 & 0.765 & $h=2$ \\
Variational & 34.41 & 0.946 & $n_{\text {iter }}=350$ & $\mathbf{2 8 . 0 3}$ & 0.782 & $n_{\text {iter }}=210$ \\
IWF & 32.40 & 0.909 & - & 26.63 & 0.764 & - \\
HWF & $\mathbf{4 3 . 0 4}$ & $\mathbf{0 . 9 7 3}$ & - & 27.31 & $\mathbf{0 . 7 9 1}$ & - \\
\hline
\end{tabular}

Table I: Quantitative comparison (PSNR \& SSIM), and optimal parameters for different methods applied to the Blocks and Kidney images with different noise levels.

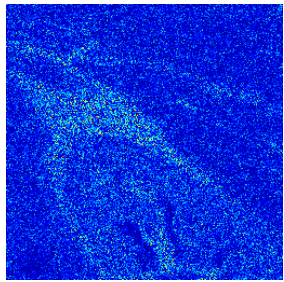

Original

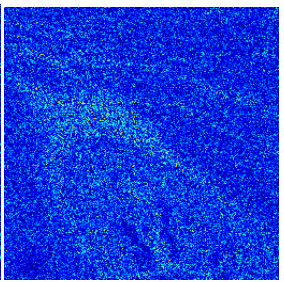

OBNLM

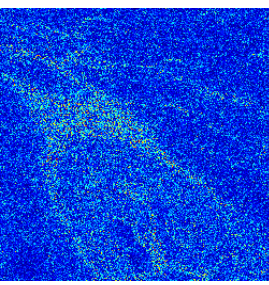

Variational

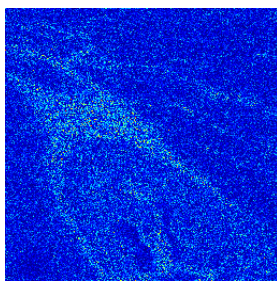

IWF

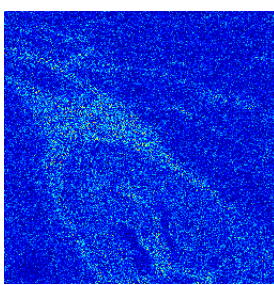

HWF

Figure 7: The method noise [22] of the various approaches applied to the Kidney image $(\sigma=3)$.

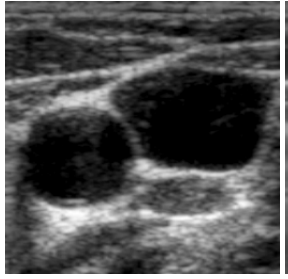

Image

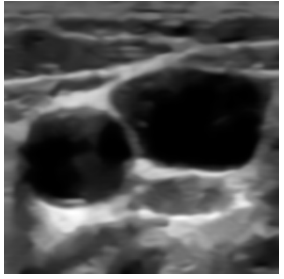

OBNLM

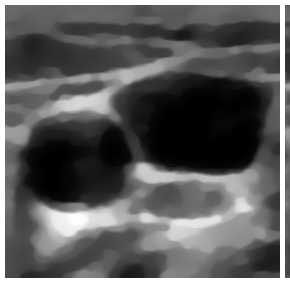

Variational

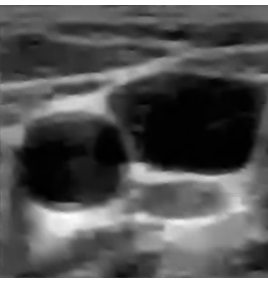

IWF

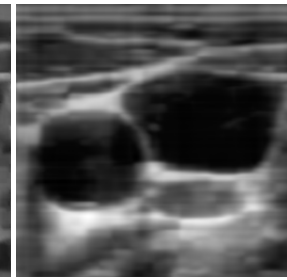

HWF

Figure 8: Visual evaluation of various methods applied to the Carotid-Thyroid image.

because the image is a piece-wise constant. However, it suffers from over-blurring effects around the edges. Artifacts due to the patching process are clearly visible in the OBNLM filter results. For the image Kidney, our approach fails at outperforming the OBNLM filter and the variational method in terms of PSNR, but remains competitive. This can be explained by the different philosophy of wavelet methods more oriented toward the complete elimination of the noise rather than the minimizing the MSE [5]. The OBNLM approach gives good results when the noise level is low. Conversely, the variational method was more appreciable for high noise levels. As can be observed in Figure 6, the wavelet approaches efficiently preserved the structure. Unfortunately, we note the presence of artifacts related to the supports of the wavelet basis. This is a common inconvenience of wavelet thresholding methods. The method noise presented in Figure 7 shows the structure of the suppressed noise for each method. The proposed method gives a good compromise between efficient suppression of the noise and preservation of structures. A major advantage of wavelet thresholding is its adaptability; the threshold comes directly from the knowledge of $\sigma$ and $\gamma$. Tuning the OBNLM filter is less straightforward, as the algorithm parameters are not directly expressed in terms of the model parameters.

2) Experiments on real data: We propose to evaluate our algorithm on some samples from real US imaging. Unfortunately, the blind extension of the SSIM presented in [37] was not suitable here as the noise is signal-dependent. Therefore comparisons and parameters tuning were entirely based on the visual quality of the resulting image. We applied the different set of parameters in Table I and choose those giving the best results. The first test concerns the Carotid-Thyroid image. In this image, the carotid 
artery, with some speckle inside due to blood flow, can be seen on the left, while the thyroid gland is visible on the right. Denoising such images may be a pre-processing step in segmentation of the thyroid gland. An enhanced image also eases the tracking of the carotid artery wall in dynamic imaging. The second test concerns Cranial US. This technique is mostly used for babies, before the cranial bones have closed, as US waves cannot pass through the skull. An example of its use is obtaining information on complications in premature birth.

Figure 8 shows results obtained using the different algorithms applied to the Carotid-Thyroid image. The results obtained using the variational method are clearly over-blurred; this is due to the piece-wise constancy constraint of total variation. The OBNLM filter achieved good results, although, there was some partitioning visible in the image. The proposed method has comparable results to those of OBNLM. Moreover, in the hyperbolic case, one can see that the horizontal structures are nicely recovered. The local treatment of the wavelets paradigm leads to images with well-defined structures. The main artifact with the proposed method is the occurrence of wavelet basis elements in the final image. The Figure 9 reveals how these artifacts can be drastically reduced when the hyperbolic wavelet is used. In the image obtained using the IWF procedure, small regions representing the supports of the Haar basis can be seen. These are similar to the artifacts related to patching that occurred using the Nonlocal methods. An improved result is obtained using the Hyperbolic settings, even though some lines are still visible.

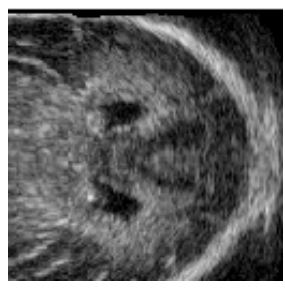

Image

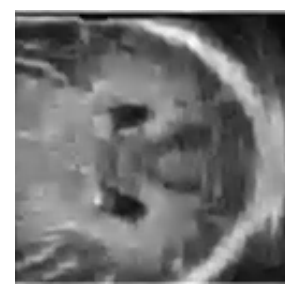

IWF

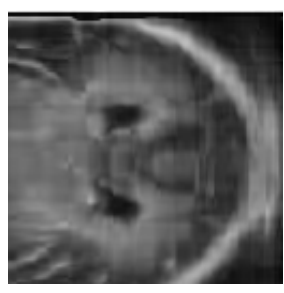

HWF

Figure 9: Visual comparison between IWF and HWF for the Brain image

\section{B. The Data-driven WF method}

In this section, our experiments reveal the potential of the data-driven extension of our algorithm. The Table II presents a comparison of the results obtained using HWF and data-driven HWF (dHWF) for the "Peppers" image studied in Figure 4. As expected, there is a loss, proportional to the noise level, in the PSNR and the SSIM up to $0.5 \mathrm{~dB}$ and $4 \%$ respectively. We believe this loss is acceptable, especially when the noise level is not very high.

\begin{tabular}{lccc|ccc}
\hline & \multicolumn{3}{c|}{ PSNR $(\mathrm{dB})$} & \multicolumn{3}{c}{ SSIM } \\
\hline$\sigma$ & 2 & 3 & 4 & 2 & 3 & 4 \\
\hline Noisy & 20.21 & 16.65 & 14.16 & 0.35 & 0.23 & 0.16 \\
HWF & 29.25 & 27.65 & 26.44 & 0.78 & 0.75 & 0.73 \\
dHWF & 29.09 & 27.40 & 26.17 & 0.76 & 0.72 & 0.69 \\
\hline
\end{tabular}

Table II: Denoising of "Peppers" image: Quantitative Comparison (PSNR \& SSIM) of the HWF and its fully data-driven version for different noise levels.

We applied this data-driven technique to the Liver image studied in Figure 3. This image has a few discontinuities, allowing the use of a large window for the mean filter. Results are given in Figure 10 for $M=8$. A first interesting result is the shape of the estimated standard deviation. A first interesting result is the "non constant slope" of the estimated standard deviation. This points out that model (1) cannot be used. We suspect that this function is proportional to the power of the image, thereby presenting an image processing based evidence of the noise model (2) relevance directly from the data. It was also noted that the set of wavelet coefficients was well stabilized. We compared the image obtained in the data-driven mode to the one obtained by using an exhaustive search for the parameter $\sigma$ with $\gamma=\{0.5 ; 1\}$. Results demonstrated that the data-driven result is satisfying and less blurred. We believe that a method free of tuning requirements is very desirable, especially for physicians.

\section{CONCLUSion}

In this paper, we described a novel approach for denoising ultrasound images based on wavelet thresholding, variance stabilization and the use of hyperbolic wavelet basis. Quantitative and visual results show the potential of the proposed method and the merit of the hyperbolic settings. A data-driven extension of our method is also presented. When applied to real data, this extension provides evidence of the relevance of the noise model. Currently, we are addressing the extension to dynamic US imaging. 
(a)
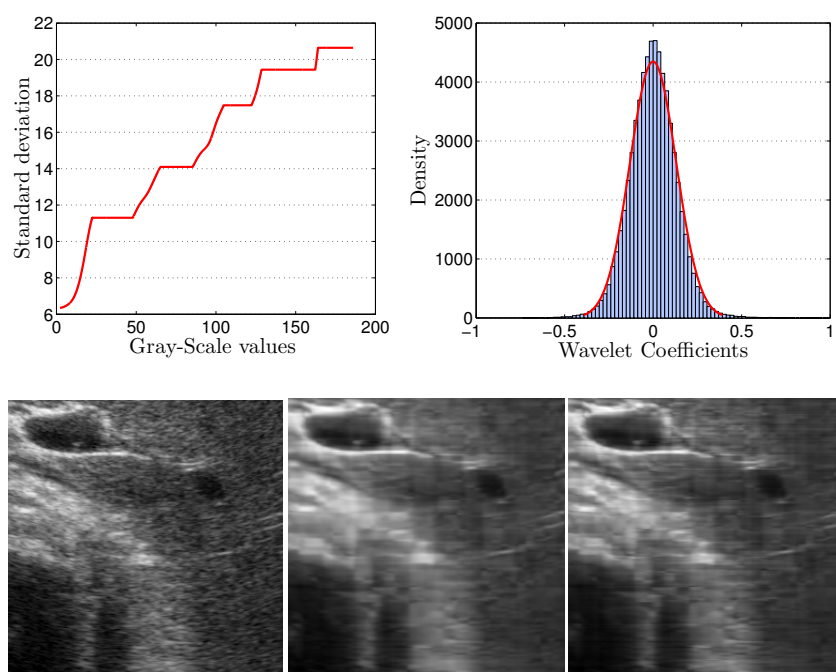

Original

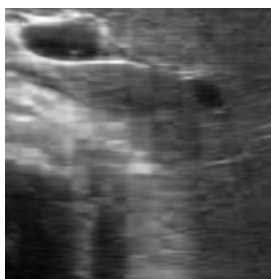

HWF

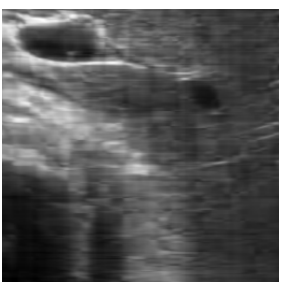

dHWF

Figure 10: Experiments on the Liver image: (a) Estimated standard deviation, (b) Blind stabilized Coefficients.

\section{APPENDIX A}

\section{VARIANCE DERIVATION}

We recall that $\eta=u^{\gamma} \varepsilon$, and we note $t=\left(t_{1}, t_{2}\right)$

$$
\begin{aligned}
\operatorname{Var}\left(\frac{d_{\underline{j}, \underline{k}}(\eta)}{\sigma \bar{u}_{\underline{j}, \underline{k}}^{\gamma}}\right) & =\frac{1}{\sigma^{2} \bar{u}_{\underline{j}, \underline{k}}^{2 \gamma}} \operatorname{Var}\left(\sum_{t} \psi_{\underline{j}, \underline{k}}(t) u^{\gamma}(t) \varepsilon(t)\right), \\
& =\frac{1}{\bar{u}_{j, \underline{k}}^{2 \gamma}} \sum_{t} \psi_{\underline{j}, \underline{k}}^{2}(t) u^{2 \gamma}(t), \\
& =\frac{1}{\bar{u}_{\underline{j}, \underline{k}}^{2 \gamma}} \sum_{t} \psi_{\underline{j}, \underline{k}}^{2}(t) u_{\underline{j}, \underline{k}}^{2 \gamma}(t) .
\end{aligned}
$$

Finally, replacing $u_{\underline{j}, \underline{k}}$ by its constant approximation $\bar{u}_{\underline{j}, \underline{k}}$ gives

$$
\operatorname{Var}\left(\frac{d_{j, \underline{k}}(\eta)}{\bar{u}_{\underline{j}, \underline{k}}^{\gamma}}\right)=\sum_{t} \psi_{\underline{j}, \underline{k}}^{2}(t)=\left\|\psi_{\underline{j}, \underline{k}, \underline{2}}\right\|_{2}^{2}=1 .
$$

\section{REFERENCES}

[1] D. Kaplan and Q. Ma, "On the statistical characteristics of the log-compressed rayleigh signals: theoretical formulation and experimental results," $J$. Acoust. Soc. Am., vol. 95, pp. 1396-1400, 1994

[2] T. Loupas, W. McDicken, and P. Allan, "An adaptive weighted median filter for speckle suppression in medical ultrasonic images," Circuits and Systems, vol. 36, pp. 129-135, 1989.

[3] P. Coupé, P. Hellier, C. Kervrann, and C. Barillot, "Nonlocal means-based speckle filtering for ultrasound images," IEEE Transactions on Image Processing, vol. 18, no. 7, pp. 2221-2229, 2009.

[4] Z. Jin and X. Yang, "A variational model to remove the multiplicative noise in ultrasound images." Journal of Mathematical Imaging and Vision, vol. 39, no. 1, pp. 62-74, 2011.

[5] D. L. Donoho and I. M. Johnstone, "Ideal spatial adaptation by wavelet shrinkage," Biometrika, vol. 81, pp. 425-455, 1994.

[6] P. Fryzlewicz, "Data-driven wavelet-fisz methodology for nonparametric function estimation," Electronic journal of statistics, vol. 2, pp. 863-896, 2008.

[7] M. H. Neumann, "Multivariate wavelet thresholding in anisotropic function spaces," Statistica Sinica, vol. 10, pp. 399-431, 2000.

[8] F. Autin, G. Claeskens, and J. Freyermuth, "Asymptotic performance of projection estimators in standard and hyperbolic wavelet bases," To appear in Electronic journal of statistics, 2015.

[9] J.-S. Lee, "Digital image enhancement and noise filtering by use of local statistics," IEEE Trans. Pattern Anal. Mach. Intell., vol. 2, no. 2, pp. 165-168, 1980.

[10] V. S. Frost, J. A. Stiles, K. S. Shanmugan, and J. C. Holtzman, "A model for radar images and its application to adaptive digital filtering of multiplicative noise," IEEE Trans. Pattern Anal. Mach. Intell., vol. 4, no. 2, pp. 157-166, 1982.

[11] D. T. Kuan, A. A. Sawchuk, T. C. Strand, and P. Chavel, "Adaptive noise smoothing filter for images with signal-dependent noise." IEEE Trans. Pattern Anal. Mach. Intell., vol. 7, no. 2, pp. 165-177, 1985.

[12] P. Perona and J. Malik, "Scale-space and edge detection using anisotropic diffusion," IEEE Trans. Pattern Anal. Mach. Intell., vol. 12, no. 7, pp. 629-639, 1990.

[13] Y. Yu and S. T. Acton, "Speckle reducing anisotropic diffusion." Image Processing, IEEE Transactions on, vol. 11, no. 11, pp. 1260-1270, 2002.

[14] K. Krissian, C. F. Westin, R. Kikinis, and K. G. Vosburgh, "Oriented speckle reducing anisotropic diffusion," Image Processing, IEEE Transactions on, vol. 16, pp. 1412-1424, 2007. 
[15] G. Ramos-Llorden, G. Vegas-Sanchez-Ferrero, M. Martin-Fernandez, and C. Alberola-Lopez, Image Processing, IEEE Transactions on, vol. 24, no. 1, pp. $345-358$.

[16] X. Zong, A. F. Laine, and E. A. Geiser, "Speckle reduction and contrast enhancement of echocardiograms via multiscale nonlinear processing," IEEE Trans. Med. Imag.

[17] A. Achim, A. Bezerianos, and P. Tsakalides, "Novel bayesian multiscale method for speckle removal in medical ultrasound images." IEEE Trans. Med. Imaging, vol. 20, no. 8, pp. 772-783, 2001.

[18] G. Samorodnitsky and M. S. Taqqu, Stable non-Gaussian random processes : stochastic models with infinite variance, ser. Stochastic modeling. New York: Chapman \& Hall, 1994.

[19] M. Suhling, M. Arigovindan, C. Jansen, P. Hunziker, and M. Unser, "Myocardial motion analysis from b-mode echocardiograms," Image Processing, IEEE Transactions on, vol. 14, no. 4, pp. 525-536, 2005.

[20] T. Tuthill, R. Sperry, and K. Parker, "Deviation from rayleigh statistics in ultrasonic speckle," Ultrason. Imag., pp. 81-90, 1988.

[21] D. Tenbrinck, S. Schmid, X. Jiang, K. P. Schäfers, and J. Stypmann, "Histogram-based optical flow for motion estimation in ultrasound imaging," Journal of Mathematical Imaging and Vision, vol. 47, no. 1-2, pp. 138-150, 2013.

[22] A. Buades, B. Coll, and J.-M. Morel, "A non-local algorithm for image denoising," in Proceedings of the 2005 IEEE Computer Society Conference on Computer Vision and Pattern Recognition (CVPR'05), vol. 2, 2005, pp. 60-65.

[23] L. Rudin, S. Osher, and E. Fatemi, "Nonlinear total variation based noise removal algorithms," Physica D, vol. 60, pp. 259-268, 1992.

[24] L. Rudin, P. L. Lions, and S. Osher, Multiplicative denoising and deblurring: theory and algorithms, ser. Geometric Level Set Methods in Imaging, Vision, and Graphics. S. Osher and N. Paragios, Eds., 2003.

[25] L. Condat, "A generic proximal algorithm for convex optimization: application to total variation minimization," Signal Processing Letters, IEEE, vol. 21, no. 8, pp. 985-989, 2014.

[26] S. Mallat, A Wavelet Tour of Signal Processing. Academic Press, 1999.

[27] S. G. Roux, M. Clausel, B. Vedel, S. Jaffard, and P. Abry, "Self-similar anisotropic texture analysis: The hyperbolic wavelet transform contribution," Image Processing, IEEE Transactions on, vol. 22, no. 11, pp. 4353-4363, 2013.

[28] N. Remenyi, O. Nicolis, G. Nason, and B. Vidakovic, "Image denoising with 2d scale-mixing complex wavelet transforms," Image Processing, IEEE Transactions on, vol. 23, no. 12, pp. 5165-5174, 2014

[29] P. Fryzlewicz and G. Nason, "A haar-fisz algorithm for poisson intensity estimation," Journal of Computational and Graphical Statistics, vol. 13 (3), pp. 621-638, 2004

[30] M. Fisz, "The limiting distribution of a function of two independent random variables and its statistical application," Colloquium Mathematicum, vol. 3 , pp. 138-146, 1955.

[31] J. M. Fadili, J. Mathieu, B. Romaniuk, and M. Desvignes, "Bayesian wavelet-based poisson intensity estimation of images using the fisz transformation," in Proc.International Conference on Image and Signal Processing, Agadir, Morocco, 2003, pp. 242-253.

[32] G. P. Nason and B. W. Silverman, "The stationary wavelet transform and some statistical applications," in Lecture Notes in Statistics. Springer-Verlag, 1995, pp. 281-300.

[33] G. Nason, Wavelet methods in statistics with R. Springer Science \& Business Media, 2010.

[34] S. Jaffard, "Beyond besov spaces part 1: Distributions of wavelet coefficients," Journal of Fourier Analysis and Applications, vol. 10, no. 3, pp. 221-246, 2004.

[35] P. Mair, K. Hornik, and J. de Leeuw, "Isotone optimization in r: pool-adjacent-violators algorithm (pava) and active set methods," Journal of statistical software, vol. 32, no. 5, pp. 1-24, 2009.

[36] Z. Wang, A. C. Bovik, H. R. Sheikh, and E. P. Simoncelli, "Image quality assessment: From error visibility to structural similarity," Image Processing, IEEE Transactions on, vol. 13, no. 4, pp. 600-612, 2004.

[37] X. Kong, K. Li, Q. Yang, L. Wenyin, and M.-H. Yang, "A new image quality metric for image auto-denoising," in Computer Vision (ICCV), 2013 IEEE International Conference on. IEEE, 2013, pp. 2888-2895. 